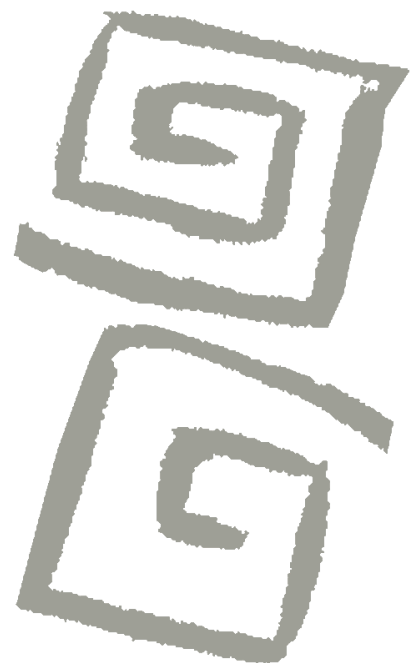

\title{
El perfil general del excedente nutrimental en México en el periodo 1990-2013: un enfoque a partir del suministro energético de macronutrimentos y grupos de alimentos
}

\author{
General profile of the nutrition surplus in Mexico from \\ 1990-2013: An approach using the energy supplied by \\ macronutrients and food groups
}

José Cutberto Hernández Ramírez ${ }^{1}$, Judith Elena Ortega Canto²

${ }^{1}$ Doctor en Ciencias de la Salud, Universidad Autónoma de Yucatán, México. $\bowtie$ (iD

${ }^{2}$ Doctora en Ciencias Sociales, con especialidad en Antropología Médica. Profesora-Investigadora jubilada, Centro de Investigaciones Regionales Hideyo Noguchi, Universidad Autónoma de Yucatán, México. $\bowtie$ (iD)
RESUMEN En este texto se analiza la evolución del suministro excesivo de kilocalorías en México desde 1990 a 2013. Para cada año del periodo se estimó el requerimiento de energía y macronutrimentos de la población mexicana y se lo contrastó con el respectivo suministro per cápita para estimar la discrepancia entre requerimiento y suministro. Las discrepancias se analizaron como serie temporal. Así, el excedente energético osciló entre 700 y 800 kcal/día per cápita en todo el periodo y los azúcares-dulcificantes aportaron el mayor suministro energético por encima de su requerimiento. El exceso de lípidos se incrementó de modo intenso y constante principalmente por el aumento de lípidos de carne de aves de corral y cerdo. El exceso aportado por las bebidas alcohólicas tendió a polarizarse en el creciente consumo de cerveza. En suma, a partir de los azúcaresdulcificantes y la carne se tendió a configurar el suministro energético y su respectivo excedente. Esto tiene implicaciones directas en la prevalencia de enfermedades crónicas no transmisibles así como en el uso insostenible de la tierra, el agua y la energía.

PALABRAS CLAVES Hipernutrición; Obesidad; Dieta Occidental; Nutrición, Alimentación y Dieta; México.

\begin{abstract}
This text analyzes the evolution of the excessive food energy supply in Mexico from 1990 to 2013. For each year, the energy and macronutrient requirements of the Mexican population were estimated and contrasted with the per capita energy supply. Discrepancies between requirement and supply were analyzed as a time series. The energy surplus ranged from 700 to $800 \mathrm{kcal}$ per capita per day throughout the studied period and sugar/sweeteners contributed the highest above-requirement energy supply. Lipids excess increased steadily and intensely, mainly due to lipid increases from poultry and pork. Excess energy from alcoholic beverages tended to be concentrated into growing beer consumption. In summary, the energy supply and the corresponding surplus tended to be made up mainly of sugar/sweeteners and meat. This has direct implications for the prevalence of chronic non-communicable diseases as well as unsustainable use of land, water and energy.
\end{abstract}

KEY WORDS Overnutrition; Obesity; Diet, Western; Diet, Food and Nutrition; Mexico. 


\section{INTRODUCCIÓN}

El engordamiento de las poblaciones humanas es un fenómeno registrado a partir de la segunda mitad del siglo XX. Según la Organización Mundial de la Salud (OMS) desde 1980 la prevalencia de obesidad se duplicó a nivel mundial y en el 2014 entre los mayores de 18 años en todo el mundo hay $13 \%$ y $39 \%$ de obesos y pre-obesos respectivamente ${ }^{(1)}$. En otras palabras, el 52\% de los individuos excede el límite del volumen corporal normal.

En ese contexto, una serie de investigaciones ha estudiado los cambios en la dieta de diversas poblaciones en el mundo durante las últimas cinco décadas. Así, se ha propuesto que en ese período en las poblaciones más obesas se incrementó la ingesta de alimentos refinados, azúcar, dulcificantes y grasas ${ }^{(2,3,4)}$.

En México, entre 1989 y 2012, la prevalencia de exceso de peso creció $^{(5,6,7)}$ y los cambios en la alimentación presumiblemente vinculados a tal fenómeno se han estudiado desde diferentes enfoques aquí clasificados como: suministro disponible per cápita; consumo alimentario autodeclarado; gasto monetario en alimentos autodeclarado y balanza comercial alimentaria.

Suministro disponible per cápita: Según este enfoque, en el periodo 1980-2000 se incrementó el suministro per cápita $-y$ por tanto el consumo aparente- de grasas animales, verduras, frutas, bebidas alcohólicas, carnes, huevo y se redujo el de leguminosas, oleaginosas, aceites vegetales y mariscos. Asimismo de 1961 a 1981 la energía diaria disponible por habitante se incrementó sostenidamente y después se mantuvo constante en poco más de $3.000 \mathrm{kcal}^{(8)}$.

Consumo alimentario autodeclarado: En las investigaciones basadas en cuestionarios de frecuencia de consumo semanal se afirma que de 1988 a 1999 se incrementó la ingesta de grasas y alimentos refinados ${ }^{(9)}$. Además se propone que, en 2006, los mexicanos que consumieron más comida rápida, refrescos, bebidas alcohólicas, pan blanco, dulces y botanas tuvieron un riesgo de obesidad de un $14 \%$ más que quienes comen una dieta más "tradicional" basada principalmente en maíz. Lo mismo ocurrió con las personas que ingirieron más lácteos, arroz, pasta, carnes, huevos, frutas y vegetales ya que presentaron un riesgo de obesidad de un $17 \%$ más ${ }^{(10)}$. Por su parte, en las investigaciones basadas en cuestionarios de recordatorio de 24 hs se afirma que, en el periodo 1988-1999, el aporte de las grasas a la ingesta energética total diaria creció del $23,5 \%$ al $30,3 \%$ mientras que el aporte relativo de los cereales y las proteínas se redujo levemente ${ }^{(5)}$.

Gasto en alimentos auto declarado: Las investigaciones basadas en las encuestas nacionales de ingresos y gastos en los hogares plantean que, entre 1984 y 1998, disminuyó el gasto total en alimentos; aunque el gasto relativo se incrementó en carbohidratos refi$\operatorname{nados}(6,3 \%)$ y refrescos $(37,2 \%)^{(5)}$. Asimismo, se incrementó el gasto monetario en cereales, alimentos preparados para consumir en el hogar, bebidas sin alcohol y alimentos consumidos fuera de casa mientras que decreció el gasto en leguminosas y carnes ${ }^{(11)}$.

Balanza comercial alimentaria: Clark et al. ${ }^{(12)}$ sostienen que con el Tratado de Libre Comercio de América del Norte la política comercial y agrícola estadounidense transformó el ambiente alimentario mexicano y que EE.UU. "exportó la obesidad" a México mediante dos vías complementarias: a) el incremento de las exportaciones de maíz, soya, azúcar y dulcificantes, carnes y productos listos para consumir, y b) la inversión creciente de capital estadounidense en toda la cadena que conforma el sistema alimentario mexicano.

Ahora bien, en los enfoques citados se describen los cambios en la dieta únicamente en términos de incrementos o decrementos, relativos o absolutos, en el consumo de ciertos alimentos, y no se describe ese consumo en términos de exceso. En otras palabras, no se especifica cuánto alimento es exceso y por qué lo es. Por ejemplo, aunque en México, de 1988 a 1999, el aporte energético diario de grasas pasó del $23,5 \%$ al $30,2 \%{ }^{(5)}$, la OMS recomienda un aporte de grasas del 15-30\% de la ingesta energética total diaria ${ }^{(13)}$; es 
decir, en ambos casos $(23,5 \%$ y $30,2 \%)$ el consumo de grasas estuvo dentro del margen recomendado.

Además, en las discusiones y/o conclusiones de los estudios pertenecientes a los primeros dos enfoques presentados no es clara la distinción entre macronutrimentos (proteínas, lípidos y carbohidratos), grupos de alimentos y alimentos concretos. Por tanto, la transmisión de resultados es más ambigua de lo que podría ser.

En efecto, aquí se propone el concepto "excedente nutrimental" entendido como: la cantidad nutricionalmente innecesaria de kilocalorías ingeridas en un periodo estipulado y cuyo aporte constante se relaciona potencialmente con la acumulación de tejido adiposo. Así, proponemos que tal concepto es útil para articular explicaciones más precisas respecto al exceso de peso en las poblaciones y su relación con las tendencias y cambios coyunturales en el ambiente alimentario, es decir, qué tipo de comida está disponible, dónde y a qué precio ${ }^{(12)}$.

Así pues, el objetivo de esta investigación es describir los cambios generales del excedente nutrimental anual en México durante el periodo 1990-2013 en dos niveles de abstracción: macronutrimentos y grupos de alimentos. Esto proporcionará elementos que contribuirán a una mejor comprensión del suministro alimentario mexicano actual y su relación con diversas enfermedades crónicas vinculadas a la sobrealimentación.

\section{MÉTODOS}

\section{Datos}

Antes de estimar el excedente nutrimental en los años comprendidos entre 1990 y 2013 fue necesario: a) registrar el suministro energético diario per cápita de macronutrimentos en cada año, y b) calcular el promedio del requerimiento energético diario per cápita de macronutrimentos de la población mexicana en cada año.

Para registrar el suministro energético diario per cápita de alimentos se consultaron las hojas de balance elaboradas por la Organización de las Naciones Unidas para la Agricultura y la Alimentación (FAO), en las que se estima el suministro alimentario disponible para el consumo humano en un país. Para ello, se suma la producción nacional más las importaciones, a las que se restan las exportaciones alimentarias, alimento para ganado, semillas, o destinado para usos no alimentarios, así como las pérdidas ocurridas por almacenamiento y transporte. La cantidad de alimento disponible para consumo humano se divide entre la población existente en un año, y se obtienen estimaciones del suministro anual de alimentos por persona ${ }^{(14)}$.

Además, se realiza la conversión de los alimentos procesados a su equivalente en productos primarios con los cuales son elaborados, tal proceso se denomina estandarización vertical. Por ejemplo, el pan es expresado en sus cantidades equivalentes de trigo, grasa vegetal y otros ingredientes utilizados para su preparación, de modo que los datos sobre disponibilidad de trigo incluyen su equivalente en trigo entero, harina de trigo y productos de harina de trigo. Asimismo, la clasificación de los productos específicos en grupos de alimentos, con sus respectivas estimaciones, se denomina estandarización horizontal. Finalmente, los datos sobre el suministro alimentario per cápita se expresan cuantitativamente y mediante factores de composición alimentaria en términos de energía, proteínas y lípidos adecuados tanto de productos primarios como de procesados.

Cabe resaltar que, en el caso de México, las hojas de balance de la FAO obtienen datos, principalmente, del Banco de Información Económica (BIE), dependiente del Instituto Nacional de Estadística y Geografía (INEGI)(15). A su vez, el BIE presenta datos del sector alimentario provenientes de las encuestas de la Secretaría de Agricultura, Ganadería, Desarrollo Rural, Pesca y Alimentación (SAGARPA), cuya estimación incluye, en el caso de maíz y trigo en granos, frijol, sorgo y arroz, la proporción no comercializable, o que no se lleva al mercado, que ciertos productores rurales usan para el 
autoconsumo $^{(16,17)}$. Pero para estimar el suministro de todos los demás alimentos, en el BIE solo se incluye la producción legal que se comercializa y paga impuestos en el circuito del mercado.

Por otro lado, para obtener el promedio anual del requerimiento energético diario per cápita de macronutrimentos de la población mexicana, se consultaron estándares antropométricos $^{(18)}$ para conocer el peso medio para la edad en grupos quinquenales por edad y sexo, y multiplicarlo por su factor correspondiente a la tasa metabólica basal por kg de peso corporal ${ }^{(19)}$. En los mayores de 10 años tal producto se multiplicó por un Coeficiente de 1,55 relativo al gasto energético por actividad física.

Posteriormente se obtuvo un promedio ponderado del requerimiento energético diario de la población mexicana en cada año estudiado según las proporciones existentes de los grupos quinquenales por edad y sexo. Para ello se usaron las estimaciones y proyecciones poblacionales del Consejo Nacional de Población ${ }^{(20)}$ de 1990 al 2013, y se añadió la revisión de los censos 1960, 1970 y 1980 del INEGI ${ }^{(21)}$.

Por último, los requerimientos energéticos de lípidos y proteínas se estimaron con base en el límite superior de los márgenes de las metas de ingestión de macronutrimentos propuestos por la $\mathrm{OMS}^{(13)}$ en términos de porcentaje de la ingesta energética total, los cuales son: lípidos $15-30 \%$ y proteínas $10-15 \%$. Respecto a la ingesta de carbohidratos, la OMS recomienda ajustar el porcentaje entre 55-75\% (incluidos azúcares con menos del 10\%) en función de la determinación previa de los requerimientos de lípidos y proteínas. Por tanto, los porcentajes de la ingesta energética total considerados excedentes energéticos de macronutrimentos fueron: lípidos $>30 \%$, carbohidratos totales $>55 \%$ (incluidos azúcares $>10 \%$ ) y proteínas $>15 \%$.

\section{Cálculo del excedente nutrimental}

El excedente nutrimental total de cada año comprendido entre 1990 y 2013 se calculó en cuatro pasos: 1) registro del suministro energético diario per cápita de macronutrimentos en el año de interés; 2) estimación de los requerimientos energéticos diarios de macronutrimentos para la población mexicana en dicho año; 3) sustracción del requerimiento energético diario de cada macronutrimento a su suministro energético diario per cápita en tal año y 4) sumar las diferencias obtenidas en el paso tres, más el aporte energético diario per cápita proveniente de las bebidas alcohólicas en el mismo año.

\section{Análisis}

El excedente nutrimental anual durante el periodo 1990-2013 se estudió como serie temporal de la cual se analizaron, tanto en términos absolutos como relativos, los cambios en los macronutrimentos que lo constituyen, así como sus respectivas variaciones anuales acumulativas y tendencias de suministro.

Se estudiaron, además, las variaciones anuales acumulativas y cambios tendenciales de los grupos de alimentos que más contribuyeron a la variabilidad en los suministros excedentes de azúcares y dulcificantes, carbohidratos y lípidos durante el periodo referido.

\section{RESULTADOS}

\section{Excesos absolutos}

Aunque el periodo de interés en este estudio es 1990-2013, en el cual se incrementó súbitamente la incidencia de exceso de peso en México, revisaremos brevemente ciertas tendencias previas del excedente nutrimental en tal país. Así, en la Figura 1 se observa cómo entre 1961 y 1980 el suministro excesivo total diario de kilocalorías creció hasta casi duplicarse y luego, de la década de 1980 a 2010, se mantuvo casi estable.

Además, durante las décadas de 1960 y 1970, el excedente nutrimental se conformó únicamente por energía de carbohidratos, azúcares-dulcificantes y bebidas alcohólicas. 


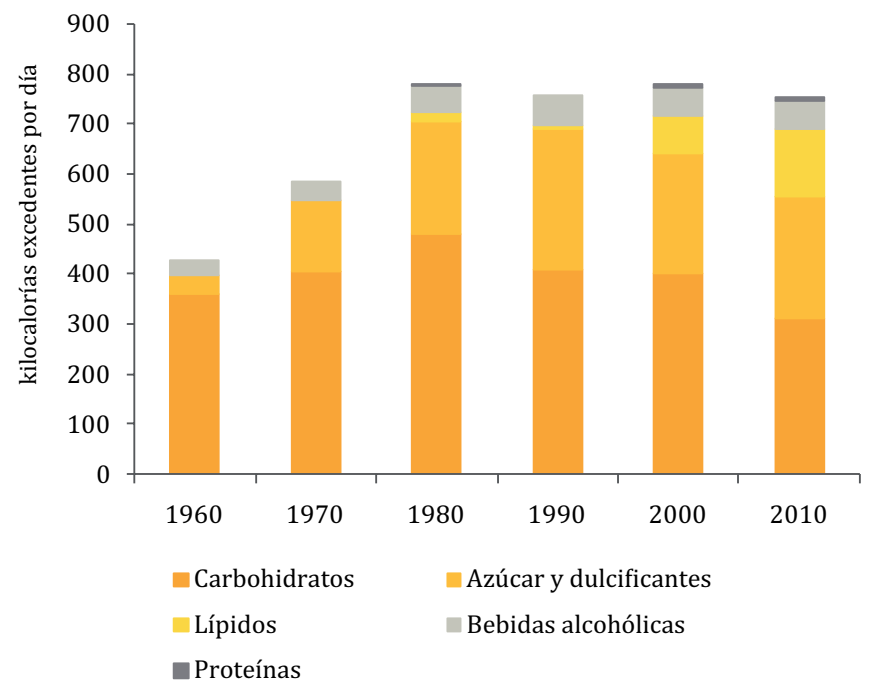

Figura 1. Cambios en el excedente energético alimentario per cápita en cifras absolutas. México, 1960-2010.

Fuente: Elaboración propia basada en datos del Consejo Nacional de Población (CONAPO) ${ }^{(17)}$ y Food and Agriculture Organization Corporate Statistical Database (FAOSTAT) ${ }^{(14)}$.

Y fue solo a partir de 1980 cuando las kilocalorías provenientes de lípidos se tornaron excesivas para incrementarse constantemente después de 1990. Respecto a las kilocalorías de proteína, se integraron definitivamente como parte del excedente nutrimental desde el año 2000 en adelante (Figura 1).

Entre 1990 y 2013 el excedente nutrimental osciló entre 700 y $800 \mathrm{kcal} / \mathrm{día}$, y en su composición proporcional desde el año 2000 se produjo la incorporación de un ligero exceso de energía proveniente de proteínas que presentó cambios irregulares -es decir, no mostró tendencia- y una variación media anual acumulativa del 1,8 \% (Figura 2). Pero el cambio más sobresaliente fue el incremento lineal del exceso energético de lípidos $\left(\mathrm{R}^{2}=0,89\right)$ y la reducción lineal del exceso proveniente de carbohidratos $\left(\mathrm{R}^{2}=0,68\right)$.

Por su parte, el aporte excesivo de azúcares y dulcificantes se mantuvo con cambios irregulares y una variación media anual acumulativa del $-0,8 \%$. Asimismo, las bebidas alcohólicas mostraron cambios irregulares y una variación media anual acumulativa del $-0,3 \%$.
En síntesis, entre las observaciones más relevantes en el período 1990-2013 se encuentran: 1) la estabilidad del excedente total entre 700 y $800 \mathrm{kcal} / \mathrm{d}$ ía aproximadamente, 2) la permanencia de azúcar-dulcificantes y bebidas alcohólicas como elementos consolidados del excedente nutrimental, y 3) se sugiere el inicio y la continuidad de una paulatina sustitución del aporte energético excesivo de carbohidratos por parte del creciente exceso de lípidos.

\section{Excesos relativos}

Entre 1990 y 2013, el exceso energético per cápita de azúcares y dulcificantes se mantuvo alrededor del doble de su requerimiento específico según la ingesta recomendada por la $\mathrm{OMS}^{(13)}$. Por tanto, puede plantearse que los azúcares y dulcificantes son el tipo de carbohidratos que se consumen más excesivamente en México. Después, los excesos de los demás carbohidratos y de los lípidos ocupan el segundo y tercer lugar de 


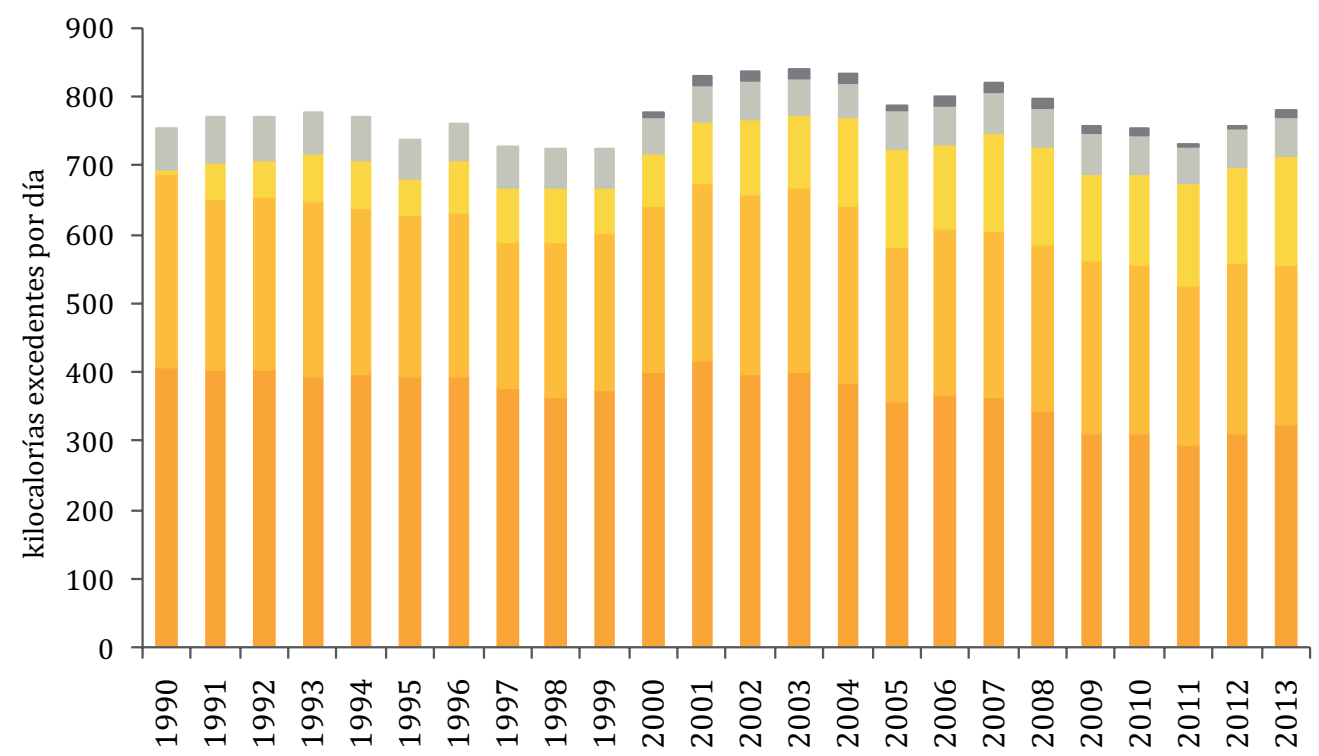

Carbohidratos $\square$ Azúcary dulcificantes $\square$ Lípidos Alcohol Proteínas

Figura 2. Cambios en el excedente energético alimentario per cápita en cifras absolutas. México, 1990-2013.

Fuente: Elaboración propia basada en datos del Consejo Nacional de Población (CONAPO) ${ }^{(17)}$ y Food and Agriculture Organization Corporate Statistical Database (FAOSTAT) ${ }^{(14)}$.

Nota: el valor 0 equivale al límite superior de la recomendación consuntiva diaria establecida por la OMS ${ }^{(13)}$ para los respectivos ítems graficados. Todo valor superior a 0 aparece como excedente energético.

importancia respectivamente mientras que las proteínas aún muestran un exceso ligero (Figura 3).

Además, los excesos energéticos relativos de azúcares-dulcificantes y proteínas mostraron variaciones irregulares, mientras que los excesos energéticos relativos de carbohidratos y lípidos sugieren una paulatina sustitución de los carbohidratos por los lípidos (Figura 3).

\section{Cambios en el suministro energético total per cápita de azucares y dulcificantes}

La energía proveniente de "azúcar equivalente"(14) (azúcar centrifugado sin refinar, azúcar refinado, azúcar para confitería y azúcares aromatizados) presentó una variación media anual acumulativa del -1,2\% de 1990 a 2013; aunque aportó en promedio el $92 \%$ del suministro energético total de azúcares y dulcificantes en tal periodo (Tabla 1).

Después del azúcar equivalente, los "dulcificantes"(14) (fructosa y maltosa químicamente puras, glucosa, dextrosa, galactosa, isoglucosa, melazas, jarabe de fructosa y azúcar invertido) aportaron en promedio el $6,8 \%$ del suministro energético total de azúcares y dulcificantes, no obstante su variación media anual acumulativa fue la mayor con un 9,9\% (Tabla 1). Más aun, puede sugerirse que la reducción del aporte de azúcar equivalente se compensó con el aumento energético de los dulcificantes.

Por su parte, el azúcar no centrifugado y la miel aportaron en promedio el $0,8 \%$ y el $0,4 \%$ del suministro energético total de azúcares y dulcificantes. Asimismo el azúcar no 


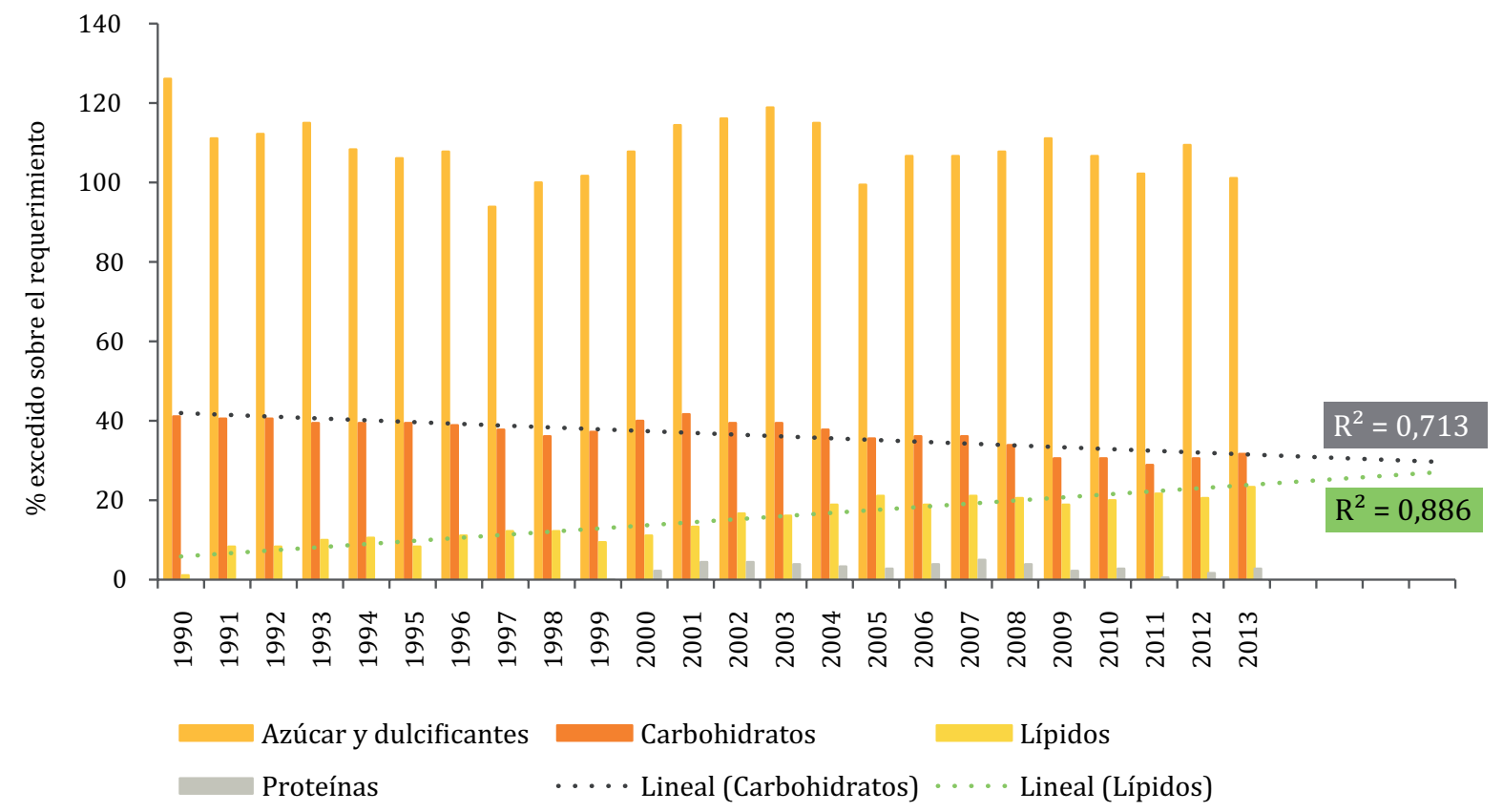

Figura 3. Cambios en los excesos relativos de macronutrimentos sobre su respectivo requerimiento. México, 1990-2013.

Fuente: Elaboración propia basada en datos del Consejo Nacional de Población (CONAPO) ${ }^{(17)}$ y Food and Agriculture Organization Corporate Statistical Database (FAOSTAT) ${ }^{(14)}$.

Nota: el valor $0 \%$ equivale al límite superior de la recomendación consuntiva diaria establecida por la OMS ${ }^{(13)}$ para los respectivos ítems graficados. Todo valor superior a 0 aparece como excedente energético.

centrifugado presentó una variación media anual acumulativa del $-2,2 \%$, mientras que la miel no mostró variaciones (Tabla 1).

En suma, el suministro energético per cápita diario de azúcares y dulcificantes en México durante el periodo 1990-2013 presentó cambios irregulares sin tendencias fuertemente definidas, y una variación media anual acumulativa del $-0,4 \%$; no obstante, tales azúcares y dulcificantes se mantuvieron como el principal exceso relativo del excedente nutrimental.

\section{Cambios en el suministro energético per cápita de carbohidratos}

En el periodo 1990-2013 se redujo el aporte energético de los carbohidratos al excedente nutrimental en México debido a que los cereales y las leguminosas disminuyeron su contribución al suministro energético total de carbohidratos; sin embargo, aun así, ambos conformaron la mayor parte de dicho suministro. De tal forma, los cereales mostraron un descenso con tendencia lineal $\left(R^{2}=0,8274\right)$ y una variación media acumulativa anual de $-0,3 \%$, mientras las leguminosas presentaron una reducción con cambios irregulares y la respectiva variación media acumulativa anual del $-0,3 \%$ (Tabla 1 ).

Cabe distinguir que la reducción del suministro energético proveniente de los cereales se debió a las disminuciones de los aportes de trigo, en primer lugar, y de maíz, en segundo; aunque ambos cereales aportaron en promedio el $93,8 \%$ de las kilocalorías de cereales. En contraste, los demás cereales presentaron incrementos, incluso la avena mostró un aumento con buen ajuste lineal $\left(\mathrm{R}^{2}=0,8011\right)$. 
Tabla 1. Proporciones promedio, variaciones y tendencias del suministro energético per cápita de macronutrimentos aportados por diversas fuentes. México, 1990-2013.

$\begin{array}{lccc}\begin{array}{l}\text { Macronutrimentos y sus } \\ \text { fuentes }\end{array} & \begin{array}{c}\text { Proporción promedio } \\ \text { en el periodo }\end{array} & \begin{array}{c}\text { Variación media } \\ \text { anual acumulativa }\end{array} & \begin{array}{c}\text { Coeficiente de } \\ \text { tendencia lineal } \\ \left(\mathbf{R}^{2}\right)\end{array}\end{array}$

\begin{tabular}{|c|c|c|c|}
\hline \multicolumn{4}{|l|}{ Azúcares y dulcificantes } \\
\hline Azúcar equivalente & 92,0 & $-1,2$ & 0,46 \\
\hline Dulcificantes & 6,8 & 9,9 & 0,62 \\
\hline Azúcar no centrifugada & 0,8 & $-2,2$ & 0,64 \\
\hline Miel & 0,4 & 0,0 & 0,03 \\
\hline Total & 100,0 & $-0,4$ & 0,01 \\
\hline \multicolumn{4}{|l|}{ Carbohidratos } \\
\hline Cereales (total) & 80,2 & $-0,3$ & 0,83 \\
\hline Maíz & 73,1 & $-0,3$ & 0,60 \\
\hline Trigo & 20,7 & $-0,6$ & 0,78 \\
\hline Arroz & 4,4 & 1,3 & 0,55 \\
\hline Avena & 0,1 & 3,3 & 0,80 \\
\hline Otros & 1,6 & 2,8 & 0,01 \\
\hline Frutas & 6,4 & 0,4 & 0,14 \\
\hline Leguminosas & 6,3 & $-0,3$ & 0,39 \\
\hline Lácteos & 3,1 & 0,1 & 0,26 \\
\hline Hortalizas & 2,1 & 0,7 & 0,38 \\
\hline Tubérculos almidonados & 1,9 & 0,2 & 0,54 \\
\hline Total & 100,0 & $-0,2$ & 0,55 \\
\hline \multicolumn{4}{|l|}{ Lípidos } \\
\hline Animales & 54,0 & 1,3 & 0,91 \\
\hline Vegetales & 46,0 & 0,5 & 0,10 \\
\hline Total & 100,0 & 0,9 & 0,92 \\
\hline \multicolumn{4}{|l|}{ Bebidas alcohólicas } \\
\hline Cerveza & 84,8 & 0,8 & 0,83 \\
\hline Bebidas alcohólicas destiladas & 14,8 & $-4,8$ & 0,81 \\
\hline Vino y bebidas fermentadas & 0,4 & - & $0,4 \%$ \\
\hline Total & 100,0 & 0,3 & 0,22 \\
\hline \multicolumn{4}{|l|}{ Proteínas } \\
\hline Proteínas vegetales & 57,0 & $-0,1$ & 0,51 \\
\hline Proteínas animales & 43,0 & 1,4 & 0,89 \\
\hline Total & 100,0 & 0,5 & 0,74 \\
\hline
\end{tabular}

Fuente: Elaboración propia basada en datos del Consejo Nacional de Población (CONAPO) ${ }^{(17)}$ y Food and Agriculture Organization Corporate Statistical Database (FAOSTAT) ${ }^{(14)}$.

\section{Cambios en el suministro energético per cápita de lípidos}

De 1990 a 2013 el incremento lineal del suministro energético per cápita de lípidos en México se debió al incremento lineal del aporte de lípidos de origen animal con variación media anual acumulativa del 1,3\%. Por su parte, los lípidos de origen vegetal presentaron cambios irregulares con variación media anual acumulativa del $0,5 \%$ (Tabla 1 ).

Ahora bien, entre los lípidos de origen animal los contenidos en carnes y huevo fueron los que más contribuyeron al 
Tabla 2. Variaciones, tendencia y proporciones promedio del aporte energético per cápita de lípidos aportados por diversas fuentes animales en el periodo 1990-2013.

$\begin{array}{lccc}\begin{array}{l}\text { Fuentes de lípidos } \\ \text { animales }\end{array} & \begin{array}{c}\text { Proporción } \\ \text { promedio en el } \\ \text { periodo }(\%)\end{array} & \begin{array}{c}\text { Variación media } \\ \text { anual acumulativa }\end{array} & \begin{array}{c}\text { Coeficiente de } \\ \text { tendencia lineal }\end{array} \\ & (\%) & \left(R^{2}\right)\end{array}$

Lípidos de origen animal

$\begin{array}{lrrr}\text { Carnes } & 49,0 & 2,4 & 0,97 \\ \text { Huevo } & 9,0 & 2,4 & 0,91 \\ \text { Lácteos } & 21,0 & 0,6 & 0,68 \\ \text { Grasas animales } & 18,0 & -0,9 & 0,38 \\ \text { Pescado y frutos del mar } & 2,0 & 0,3 & 0,09 \\ \text { Vísceras } & 1,0 & -0,2 & 0,41 \\ \text { Total } & 100,0 & 1,3 & 0,91\end{array}$

\section{Lípidos de carnes}

$\begin{array}{lrrr}\text { Cerdo } & 51,6 & 1,5 & 0,93 \\ \text { Aves de corral } & 31,7 & 4,5 & 0,97 \\ \text { Vaca } & 14,2 & 0,5 & 0,21 \\ \text { Ovina y caprina } & 2,0 & 0,6 & 0,13 \\ \text { Otra } & 0,5 & -2,1 & 0,78 \\ \text { Total } & 100,0 & 2,4 & 0,97\end{array}$

Fuente: Elaboración propia basada en datos del Consejo Nacional de Población (CONAPO) ${ }^{(17)}$ y Food and Agriculture Organization Corporate Statistical Database (FAOSTAT) ${ }^{(14)}$.

incremento energético mencionado, ambos con una variación media anual acumulativa del $2,4 \%$ y una fuerte tendencia lineal de crecimiento expresada en un coeficiente $\mathrm{R}^{2}$ cercano a 1 (Tabla 2). Después, en tercer lugar aparecen los lípidos de lácteos, aunque lo hacen con una variación media anual acumulativa menor y una tendencia lineal con menos fuerza de asociación entre tiempo e incremento.

En términos proporcionales, los lípidos de las carnes aportaron, en promedio, el $49 \%$ del total de lípidos de origen animal. En segundo lugar, se encuentran los lácteos con el $21 \%$ y, en tercero y cuarto, las grasas animales y el huevo con promedios del $18 \%$ y del $9 \%$, respectivamente. Por su parte, el aporte lipídico de pescados y frutos de mar, así como de las vísceras fue irrelevante, tanto en términos proporcionales como en términos de contribución al incremento lineal observado en los lípidos de origen animal durante el periodo de estudio (Tabla 2).

Pero entre las carnes cabe distinguir las que contribuyeron al incremento lineal de lípidos de origen animal. Así, fueron los lípidos provenientes de las aves de corral los que presentaron la mayor variación anual acumulativa con un $4,5 \%$ y una fuerte asociación lineal tiempo-incremento $\left(\mathrm{R}^{2}=0,97\right)$. Después de las aves de corral, el cerdo presentó una variación anual acumulativa del $1,5 \%$ y una fuerte asociación lineal tiempoincremento $\left(\mathrm{R}^{2}=0,93\right)$. Asimismo, ambos 
tipos de carne aportaron juntas el $83,3 \%$ del promedio total de lípidos provenientes de carnes en México durante el periodo estudiado (Tabla 2).

En resumen, el crecimiento de la proporción de lípidos en el excedente nutrimental entre 1990 y 2013 se debió al incremento lineal del suministro de lípidos de origen animal y, específicamente, a los aportados por carne de aves de corral y de cerdo en primer lugar, seguidos por los provenientes de huevo $y$, finalmente, por los de lácteos.

\section{El exceso energético proveniente de las bebidas alcohólicas}

El alcohol no es un alimento necesario dentro del requerimiento nutrimental de la población mexicana. En efecto, el 100\% de la energía aportada por esta bebida se consideró innecesario y, por tanto, excedente.

Así, en el periodo 1990-2013, la proporción de bebidas alcohólicas en el excedente nutrimental se redujo, con una variación media anual acumulativa del -0,3\% y cambios irregulares; pero tal reducción se debió solo a la disminución lineal $\left(\mathrm{R}^{2}=0,81\right)$ del aporte de bebidas destiladas ya que, en contraste, el aporte energético de la cerveza, además de ser el mayor, se incrementó con su respectiva variación del $0,8 \%$ y una tendencia lineal con valor $\mathrm{R}^{2}=0,83$ (Tabla 1 ).

\section{DISCUSIÓN}

La información aportada en esta investigación presenta tanto convergencias como divergencias con los planteamientos propuestos en otros estudios. A continuación se discuten tales concordancias y contrastes en torno a cuatro elementos: 1) tamaño del excedente nutrimental, 2) cambios en los aportes de macronutrimentos, 3) el excedente nutrimental y la industria de la alimentación y 4) el enfoque del suministro alimentario frente a las encuestas de alimentación.

\section{El tamaño del excedente nutrimental}

En México, desde 1961, el suministro de $\mathrm{kcal} / \mathrm{d}$ ía supera el requerimiento de la población; aunque debe distinguirse que de 1961 a 1980 tal exceso se incrementó de manera constante hasta casi duplicarse mientras que, entre 1980 y 2013, se mantuvo estable. Tales tendencias fueron descritas en un estudio en el que se plantea, sin profundizar, la "discrepancia" entre suministro y requerimiento(8). Precisamente, nosotros profundizamos en ello al formular el concepto "excedente nutrimental" y su respectivo análisis.

Pero la estimación del requerimiento energético promedio de la población mexicana realizada en esta investigación es un $27 \%$ mayor que la hecha por Ortiz et al. ${ }^{(8)}$ $y$, por lo tanto, el tamaño de tal "discrepancia" o "excedente nutrimental"-en nuestros términos- fue menor. En ese sentido, nuestra estimación presenta las siguientes características y diferencias:

- Se basa en la Convención de Roma ${ }^{(19)}$, la cual, a la luz de más evidencias científicas que su homóloga de Ginebra ${ }^{(22)}$-usada por Ortiz et al.-, presenta modificaciones en los requerimientos de niños y adolescentes.

- Para calcular el requerimiento energético de mayores de 18 usamos un factor de 1,55 que correponde al promedio para actividad física sedentaria. En contraste, según la Convención de Roma, el factor de 1,4 -usado por Ortiz et al.- es más adecuado para estimar requerimientos energéticos en intervenciones nutricias de corta duración dirigidas a personas dependientes con inactividad total y en condiciones de crisis.

- Las referencias antropométricas de peso para la edad que utilizamos están mucho más desagregadas por grupos de edad; por consiguiente, el cálculo del requerimiento es más específico para cada grupo de edad en función de su peso promedio estándar.

Ciertamente, a excepción de Ortiz et al. ${ }^{(8)}$, en algunas de las investigaciones más relevantes sobre la evolución de los patrones alimentarios en México, se obvia tal 
"discrepancia" y únicamente se describen los cambios porcentuales en la distribución de macronutrimentos en la dieta ${ }^{(9,5)}$. Incluso, en algunas de ellas, basadas en recordatorios de 24 horas, se propone que la ingesta promedio diaria de las mexicanas mayores de 18 años en 1999 fue de $1.636 \mathrm{kcal}$ diarias ${ }^{(5)}$; mientras la $\mathrm{FAO}^{(14)}$, en contraste, reportó que en tal año el suministro energético per cápita en México fue de 2.982 kcal diarias; es decir, casi el doble.

Ahora bien, como se comentó más arriba, con excepción de los suministros de maíz y trigo en granos, frijol, sorgo y arroz en México, en los cuales se consideran tanto el consumo comercializado como el autoconsumo, para todos los demás suministros calculados respecto a tal país en las hojas de balance de la FAO solo se considera la producción legal que se comercializa y paga impuestos. Esto puede conllevar al subregistro del autoconsumo y la comercialización no registrada o "informal" de frutas, hortalizas y oleaginosas, así como de la caza y la pesca de variedades distintas a las domesticadas y que no se incluyen en las categorías del catálogo de registro de información pecuaria en las encuestas mexicanas.

En efecto, es posible sugerir que el valor total del excedente nutrimental puede ser aún mayor a nuestra estimación, especialmente, por un subregistro de la energía proveniente de proteínas y grasas aportadas por ciertos productos pecuarios y oleaginosas, carbohidratos de frutas y hortalizas así como por los tipos de bebidas alcohólicas que no tienen control fiscal o sanitario. Asimismo, respecto al azúcar de caña, los datos más recientes obtenidos mediante información satelital e instrumentos de recolección mejorados muestran que, al menos en 2014 , del $100 \%$ de la producción nacional de caña de azúcar solo el 3,5\% se destina al autoconsumo.

Otra fuente de posible subregistro puede ser la discrepancia entre lo que los productores declaran producir (cifra normalmente usada para el cálculo del suministro de alimentos) y la producción real; sin embargo, hasta ahora en México no se cuenta con estudios que demuestren la existencia de tal discrepancia e incluso en los datos de 2012 en adelante la Encuesta Nacional Agropecuaria (ENA) implementa un método de verificación satelital de la superficie sembrada para cotejar lo declarado en las entrevistas. Esto último es importante para garantizar una mayor precisión de los datos a partir de tal año.

Así las cosas, si se consideran las posibles fuentes de subregistro mencionadas, el tamaño del excedente nutrimental propuesto en este artículo -de por sí ya mayor a los excesos energéticos reportados en todas las investigaciones precedentes- podría ser todavía más grande.

\section{Cambios en los aportes de macronutrimentos}

Tanto el método basado en el suministro alimentario como el apoyado en encuestas de consumo de alimentos autodeclarado coinciden en un punto: la ingesta de grasas en la población mexicana se incrementó de manera constante durante la última década del siglo XX. Más aún, en esta investigación, basada en el suministro alimentario per cápita, se evidencia que, hasta el año 2013, persistió un aumento lineal del aporte excesivo de lípidos tendiente a sustituir el exceso provisto por los carbohidratos.

Además, nuestros resultados muestran que el suministro per cápita de azúcares y dulcificantes en México es, por mucho, el que más excede las recomendaciones dietéticas de la $\mathrm{OMS}^{(13)}$; sin embargo, en los estudios basados en encuestas de consumo alimentario autodeclarado el análisis de la ingesta de azúcares es escaso y unidimensional. Escaso porque solo alude al azúcar indirectamente al comentar que se incrementó el consumo de carbohidratos refinados ${ }^{(5)}$, sin ir más allá. Unidimensional, porque se enfoca en el consumo de bebidas azucaradas $^{(23)}$ las cuales, incluidas como dulcificantes en las estimaciones de la $\mathrm{FAO}^{(14)}$, representan menos del $6,8 \%$ del suministro total de azúcares y dulcificantes mientras el $92 \%$ proviene de otras fuentes.

Así, dichas investigaciones resaltan el incremento de lípidos en la dieta y tienden 
a subregistrar y minimizar el consumo de azúcar en México y, tal vez, ese énfasis en los lípidos se debe a su coincidencia temporal con el aumento de obesidad en la población. Ciertamente, el suministro excesivo de azúcar y dulcificantes antecede por casi 10 años al primer registro de dicho aumento de obesidad y lo mismo ocurre con los excesos de carbohidratos y bebidas alcohólicas. Entonces, parece ser que, en 1990, cuando los lípidos fueron incluidos entre los excesos y comenzaron a sustituir a los carbohidratos, la obesidad comenzó a incrementarse; sin embargo, es importante advertir el peso cuantitativo y constante del azúcar y las bebidas alcohólicas en el excedente alimentario y su posible efecto aditivo a partir de la inclusión del exceso de lípidos.

Finalmente, según la teoría de la transición nutricional ${ }^{(2)}$, el perfil actual del estado nutricio de la población mexicana se correspondería, entre otros elementos, con una reducción del consumo de cereales no "industrializados". Sin embargo, Ortiz et al. ${ }^{(8)}$ afirman que el consumo alimentario en México no se apega a tal planteamiento ya que allí la disponibilidad de cereales no solo no se redujo, sino que se incrementó, con aumentos de trigo y arroz. Pero tal afirmación es muy apresurada porque obvia una tendencia: tanto desde la década de 1980 como en el periodo estudiado, el suministro de cereales $y$, especialmente, el de trigo, presenta un descenso lineal (Tabla 1). Así, nuestro estudio muestra cómo el uso de algunos elementos del análisis de series temporales permite realizar observaciones menos superficiales de los datos del suministro de alimentos.

\section{El excedente nutrimental y la industria de la alimentación}

El aumento del suministro per cápita de lípidos en México -y del excedente de lípidos- desde 1990 se debe a la expansión de la industria avícola seguido de la industria porcina. De ahí que los crecimientos lineales de los lípidos de carne de aves de corral, huevo y cerdo presenten las mayores variaciones medias anuales. $Y$ dichos datos ejemplifican ciertas tendencias internacionales de la industria cárnica, tales como la proyección de que para el 2022 casi la mitad de la carne adicional consumida en el mundo será de pollo; o bien que actualmente solo crece el mercado para productos avícolas y cerdo porque ambas especies usan comederos y pueden criarse en espacios confinados, lo cual permite satisfacer la creciente demanda de carne barata ${ }^{(24)}$.

En efecto, el incremento desde los años ochenta en las exportaciones de maíz amarillo y soja de EE.UU. hacia México ${ }^{(12)}$ corresponde a la demanda creciente de alimento para aves y cerdos por parte de tales industrias cárnicas. Por ende, esas exportaciones son correlato del incremento excesivo de lípidos de origen animal en la dieta mexicana.

Por otro lado, el exceso promedio per cápita de $243 \mathrm{kcal} /$ día de azúcar y dulcificantes entre 1990 y 2013 equivale a un peso neto de $58 \mathrm{~g}$ diarios ${ }^{(25)}$. Considérese que esos 58 g extra pueden consumirse en diversas formas ya sea como azúcar "de mesa", sacarosa integrada en alimentos dulces y salados procesados industrialmente o dulcificantes usados en la industria alimentaria para modificar las propiedades organolépticas (color, olor, sabor y textura) de los alimentos. Dado que son útiles para postergar la caducidad de múltiples productos alimenticios "industrializados" y/o potenciar su atractivo al paladar, los azúcares y dulcificantes forman parte de la dieta cotidiana aunque a veces "no se vean" ni se perciban conscientemente.

Así pues, en el perfil general del excedente nutrimental en México entre 1993 y 2013 resaltan, principalmente, el creciente exceso de lípidos provenientes de la carne producida industrialmente y el exceso relativo mayoritario y consolidado de azúcares-dulcificantes. Tales hechos coinciden con cierta hipótesis según la cual, así como los cereales fueron el centro de los sistemas alimentarios de las grandes civilizaciones del pasado (el arroz de la civilización china, el trigo de la persa y griega, el maíz de la mesoamericana), el núcleo alimentario de la sociedades industriales contemporáneas se 
conforma elípticamente a partir de dos focos que configuran el sistema alimentario: azúcar refinada y carne ${ }^{(26)}$.

Después de los azúcares-dulcificantes y los lípidos, la creciente cantidad de energía proveniente de la cerveza proyecta su propia permanencia relevante en el excedente nutrimental, mientras que el aporte energético de las bebidas destiladas tiende a la baja (Tabla 1). Y tales cambios en la composición del suministro de bebidas alcohólicas en México son análogos a los reportados en un estudio que abarcó el período 1970-1989(27); sin embargo, los datos relativos a las bebidas sin control fiscal o sanitario no se registran en las hojas de balance alimentario de la FAO, por lo tanto, existe un subregistro en el consumo total de alcohol.

Respecto al aumento del consumo de cerveza en México cabe agregar que, a finales del siglo XIX, la cerveza era un bien suntuario importado de Alemania, EE.UU. e Inglaterra y consumido por las comunidades de extranjeros en el país; sin embargo, en la primera mitad del siglo XX la cerveza mexicana desplazó a su homóloga importada y pasó de ser un bien suntuario a una bebida popular ${ }^{(28)}$. Así, México es hoy el mayor exportador mundial de cerveza ${ }^{(29)} y$, desde 1960, el capital productor de cerveza en ese país tiende a concentrarse oligopólicamente en empresas que controlan desde la producción de insumos hasta la distribución ${ }^{(30)}$.

\section{Suministro alimentario versus encuestas dietéticas}

La pertinencia de los datos de suministro alimentario de las hojas de balance de la FAO para deducir el consumo de macronutrimentos y grupos de alimentos de una población depende del nivel de análisis. Si se trata de una población nacional, entonces esos datos son un indicador imprescindible que ofrece información fiable. Pero su homogeneidad impide transpolar ipso facto tales deducciones hacia subgrupos - por edad, sexo o área geográfica- de dicha población y es allí donde el suministro alimentario puede complementarse con encuestas de alimentación.

Los métodos de encuesta comúnmente usados (recordatorio de 24 horas y frecuencias de consumo) ofrecen información más desagregada respecto al tipo de alimentos concretos que las personas dicen consumir y permiten hacer clasificaciones por edad, sexo y área geográfica. Pero tal información es útil hasta cierto punto, ya que habría que considerarla con precaución si carece de verificación empírica más allá del registro escrito de los hábitos alimentarios.

Y he ahí la cuestión, los recordatorios de 24 horas o las frecuencias de consumo son discursos codificados y no datos "empíricos", aunque se los presenta como tales, esto es: registran lo que las personas dicen que comen y no necesariamente lo que realmente comen. Entonces, tales encuestas son discursos que requieren contrastarse con las respectivas conductas que refieren $y$, de no hacer tal contraste, se corre el riesgo de confundir los discursos con las conductas y hacer una generalización sesgada por sobreregistro o, más frecuentemente, por subregistro. En tales términos, una de las principales desventajas de las encuestas es el sesgo en la cantidad de alimentos consumida; no obstante, en algún estudio se propone algo parcialmente opuesto, es decir, que las encuestas dietéticas proporcionan información detallada especialmente respecto al tamaño de las porciones ${ }^{(31)}$.

Para ejemplificar tal contradicción considérese que en EE.UU. cierta encuesta nacional registró solo el $50 \%$ del alcohol consumido según los registros de venta de bebidas alcohólicas ${ }^{(32)}$; o bien que, mientras aquí mostramos cómo el suministro de azúcares y dulcificantes en México es el más excesivo en términos relativos, en un estudio nacional reciente basado en encuestas se afirma que el $72 \%$ de la población mexicana ingiere la cantidad adecuada de azúcar y que el $80,4 \%$ declaró haber comido azúcares y dulces al menos uno de los tres días que abarcó un cuestionario tipo recordatorio de 24 horas $^{(33,34)}$.

Por otro lado, respecto a las encuestas de gasto monetario en alimentos conviene 
tener presente que son útiles para registrar los cambios y tendencias no en la dieta sino en el gasto monetario en comida. De otra forma se corre el riesgo de asimilar -o confundir- el gasto con el consumo y llegar a conclusiones erróneas, tales como decir que durante los últimos treinta años en México se ha reducido la ingesta de carnes porque en los hogares se gasta menos dinero en su compra ${ }^{(35)}$; no obstante, el suministro casi se duplicó en tal periodo ${ }^{(14)}$. Pero resulta que también los datos sobre el suministro alimentario son calculados con base en diversas encuestas, es decir, se basan en declaraciones de diversos actores sociales y no necesariamente en el dato empírico directo referido por dichos actores.

Así las cosas, no se trata de invalidar la posibilidad de acercarse a la realidad mediante las encuestas sino de señalar sus límites, resaltar la necesidad de contrastar diversas encuestas y, cuando es posible, verificar el dato empírico referido por los entrevistados. Ahora bien, la elección de datos de suministro alimentario o de encuestas dietéticas depende, entonces, de qué es lo que se desea saber y del nivel de análisis. Por ejemplo, proponemos que, por un lado, los tipos de encuesta dietética citados son útiles para responder qué se come y/o con qué frecuencia, tanto en poblaciones como en subgrupos poblacionales $y$, por otro, los datos de suministro alimentario sirven para responder cuánto se come en términos de macronutrimentos y grupos de alimentos en una población nacional y los cambios de esas cantidades a través del tiempo. Por tanto, no son métodos intercambiables, aunque sí son complementarios y, como toda indagación científica en alimentación, cada uno por separado o incluso ambos combinados, solo nos brindan un saber aproximado y parcial. En efecto, es recomendable contrastar los aportes de las encuestas dietéticas con otras fuentes de información cualitativas (observación participante) y, en el caso de poblaciones nacionales, cuantitativas (suministro de alimentos).

\section{Dieta sostenible y excedente nutrimental en México}

Según un informe de la $\mathrm{FAO}^{(36)}$ las dietas sostenibles son:

\begin{abstract}
...aquellas dietas con bajo impacto ambiental que contribuyen a la seguridad alimentaria y nutrimental y a una vida sana de las generaciones presentes y futuras. Las dietas sostenibles son protectoras y respetuosas de la biodiversidad y de los ecosistemas, son culturalmente aceptables, accesibles, económicamente justas y asequibles; nutricionalmente adecuadas, inocuas y saludables; a la vez que optimizan los recursos naturales y humanos.
\end{abstract}

Ahora bien, en México, el mayor consumo de alimentos procesados industrialmente conllevó una ingesta excesiva de azúcares-dulcificantes y grasas incluidas en carnes de pollo y cerdo. Así, tanto la diversificación de los azúcares-dulcificantes potencialmente adictivos incluidos en múltiples alimentos como el incremento excesivo de lípidos aportados por tales carnes detonaron la sobrealimentación y, por tanto, la obesidad, patologías metabólicas y cardiovasculares. Además, el suministro creciente de productos de la industria avícola y porcina incrementa el consumo de piensos transgénicos cultivados con herbicidas potencialmente nocivos (por ejemplo, el glifosato) para el ganado y los consumidores de carne ${ }^{(24)}$.

Pero hay más, producir un gramo de proteína animal requiere en promedio ocho gramos de proteína vegetal. Por consiguiente, cada gramo extra de proteína de aves y cerdo producidos intensivamente incrementa ocho veces la demanda de cereales y soja $y$, por ende, el mayor uso de tierra destinada a cultivos que satisfacen indirectamente la creciente demanda de carne por parte de los consumidores. Esto conlleva consecuencias adversas para la biodiversidad ya sea por la deforestación para destinar tierras al cultivo, por el uso de semillas transgénicas o ambas. 
Por su parte, ligado a lo anterior, la producción industrial de carne implica el uso excesivo y la contaminación del agua. Por ejemplo, mientras producir 1 kilo de papas o trigo requiere alrededor de 150 y 1.400 litros de agua respectivamente, producir un kilo de pollo requiere 4.000 litros de agua ${ }^{(37)}$. Asimismo, la sobrefertilización del suelo ocasionada por el exceso de abonos líquidos y estiércol deriva en la filtración de fósforo y nitratos hacia aguas subterráneas, ríos y lagos, pozos y manantiales, esto es: daños al suelo y la biodiversidad así como un mayor riesgo de cáncer por parte de quienes beben esa agua contaminada ${ }^{(24)}$.

A tales efectos sanitarios y ambientales derivados del consumo creciente de carnes, cabe añadir que su producción intensiva genera casi un tercio de los gases de efecto invernadero global| ${ }^{(24)}$ y que las emisiones indirectas de $\mathrm{CO}_{2}$ ocasionadas por una dieta basada en vegetales son menores que las de una dieta mixta con vegetales y productos de origen animal(38).

Con todo, así es como, según tal concepto de "dieta saludable", el suministro alimentario en México caracterizado por excesos de azúcares-dulcificantes y grasas de origen animal así como por el crecimiento lineal intenso del consumo de carne, leche y huevo, y el decrecimiento del consumo de maíz y frijol, corresponde a una dieta que carece de inocuidad, es inadecuada para los requerimientos de la población y, por ende, no contribuye a la seguridad nutricional ni a una vida sana de las generaciones presentes y futuras. No es una dieta protectora y respetuosa de la biodiversidad ni de los ecosistemas y tampoco optimiza los recursos naturales y humanos.

\section{CONCLUSIONES}

Aunque las hojas de balance de la FAO posiblemente subregistren el autoconsumo y la producción circulante en mercados "informales" que están al margen del fisco en México, vale decir que, desde hace siete décadas, en ese país la proporción de productores-autoconsumidores es cada vez menor debido a fenómenos tales como la migración del campo a la ciudad o a EE.UU. Junto a esto, la introducción creciente del dinero y diversas mercancías -incluidos los alimentos procesados industrialmente- en las comunidades rurales durante las últimas cuatro décadas las ha integrado paulatinamente en el circuito de consumo mercantil capitalista. En breve, las personas consumen cada vez menos alimentos producidos por ellas mismas y la producción agropecuaria y de alimentos, en general, tiende a concentrarse en grandes productores ${ }^{(39,40)}$, esto es: cada vez hay menos espacios que den cabida a los subregistros referidos arriba. Así pues, por las tendencias histórico-económicas en México (integración creciente de la economía rural al circuito mercantil capitalista), el indicador suministro alimentario provee información suficientemente confiable (por supuesto mejorable) respecto a los cambios generales de la alimentación en dicho país.

Los cambios en el perfil general del excedente nutrimental en México en el periodo 1990-2013 son correlato de la paulatina consolidación de un sistema alimentario industrial configurado a partir de dos focos: azúcares-dulcificantes y carne. Esto conlleva potenciales efectos adversos tanto para la salud de los consumidores de alimentos como para el medio ambiente. En efecto, la dieta mexicana en el periodo de estudio fue insustentable y tiende a mantenerse así.

Mientras el excedente nutrimental descripto en este trabajo exista, posiblemente el perfil epidemiológico de la población mexicana seguirá presentando una alta y creciente prevalencia de enfermedades crónicas no transmisibles relacionadas con la sobrealimentación, tales como diabetes, enfermedades cardiovasculares y obesidad, entre otras. $\mathrm{Ni}$ qué decir respecto al deterioro que el incremento lineal del consumo de carne industrial implicará en la contaminación del agua por nitratos -asociados con el cáncer- y en los riesgos sanitarios, ecológicos y económicos resultantes de la producción-consumo masivo de piensos 
elaborados con soya y maíz transgénico, que son riesgos: 1) sanitarios, por la exposición a sustancias tales como el herbicida glifosato; 2) ecológicos, por la reducción de la biodiversidad; y 3) económicos, por la concentración, centralización y acaparamiento de la producción alimentaria por parte de los propietarios de ciertos paquetes tecnológicos de producción.

\section{AGRADECIMIENTOS}

La investigación que derivó en este artículo recibió el apoyo del Consejo Nacional de Ciencia y Tecnología de México, a través de la beca de estudios de posgrado $N^{\circ} 338078$ otorgada al autor durante el período 2012-2015, quien a su vez fue dirigido por la autora.

\section{REFERENCIAS BIBLIOGRÁFICAS}

1. Organización Mundial de la Salud (OMS). Obesidad y sobrepeso: Nota descriptiva No 311 [lnternet]. 2015 [citado 13 ago 2016]. Disponible en: http://tinyurl.com/zmtc8u4.

2. Popkin BM. Urbanization, lifestyle changes and the nutrition transition. World Development. 1999;27(11):1905-1916.

3. Popkin BM, Nielsen CJ. The sweetening of the world's diets. Obesity Research. 2003;11(11):1325-1332.

4. Drewnowsky A, Popkin B. The nutrition transition: New Trends in the Global Diet. Nutrition Reviews. 1997;55(2):31-43.

5. Rivera JA, Barquera S, Campirano F, Campos I, Safdie M, Tovar V. Epidemiological and nutritional transition in Mexico: rapid increase of noncommunicable diseases and obesity. Public Health Nutrition. 2002;5(1A):113-122.

6. Sanchez-Castillo CP, Pichardo-Ontiveros E, Lopez-R P. Epidemiología de la obesidad. Gaceta Médica de México. 2004;140(2):3-20.
7. Gutiérrez JP, Rivera-Dommarco J, Shamah-Levy T, Villalpando-Hernández S, Franco A, Cuevas-Nasu L, Romero-Martínez M, Hernández-Ávila M. Encuesta Nacional de Salud y Nutrición 2012: Resultados Nacionales. Cuernavaca, México: Instituto Nacional de Salud Pública; 2012.

8. Ortiz-Hernández L, Delgado-Sánchez G, Hernández-Briones A. Cambios en factores relacionados con la transición alimentaria y nutricional en México. Gaceta Médica de México. 2006;142(3):181-193.

9. Rivera JA, Barquera S, González-Cossío T, Olaíz G, Sepúlveda J. Nutrition transition in Mexico and in other Latin American countries. Nutrition Reviews. 2004;62(7 Pt 2): S149-S157.

10. Flores M, Macías N, Rivera M, Lozada A, Barquera S, Rivera-Dommarco J, Tucker KL. Dietary patterns in Mexican adults are associated with risk of being overweight or obese. Journal of Nutrition. 2010;140(10):869-873. doi: 10.3945/ jn.110.121533.

11. Aboites G, Félix G. Patrones de consumo alimentario: Una visión de género. En: Aboites G, (coord). Patrones de consumo alimentario en México: retos y realidades. México: Trillas; 2011. p.139-174.

12. Clark SE, Hawkes C, Murphy SME, Hansen-Kuhn KA, Wallinga D. Exporting obesity: US farm and trade policy and the transformation of the Mexican consumer food environment. International Journal of Occupational and Environmental Health. 2012;18(1):53-65.

13. Organización Mundial de la Salud. Dieta, nutrición y prevención de enfermedades crónicas (Serie Informes Técnicos No. 797). Ginebra: OMS; 2001. 
14. Organización de las Naciones Unidas para la Alimentación y la Agricultura. Balances alimentarios [Internet]. 2015 [citado 19 mar 2016]. Disponible en: http://tinyurl.com/jg98kqp.

15. Instituto Nacional de Estadística y Geografía. Banco de Información Económica [Internet]. INEGI [citado 19 ago 2016]. Disponible en: http://tinyurl. com/hgkdyz6.

16. Secretaría de Agricultura, Ganadería, Desarrollo Rural, Pesca y Alimentación. Diseño Conceptual de la Generación de Información Agropecuaria [Internet]. 2013 [citado 19 ago 2016]. Disponible en: http://tinyurl.com/gp9rcmc.

17. Instituto Nacional de Estadística y Geografía. Encuesta Nacional Agropecuaria (ENA): Información relevante. [Internet]. 2015 [citado 19 ago 2016]. Disponible en: http://tinyurl.com/ h5y8jw9.

18. Frisancho AR. Anthropometric standards: An interactive nutritional reference of body size and body composition for children and adults. Ann Arbor: The University of Michigan Press; 2008.

19. Food and Agriculture Organization of the United Nations. Human Energy Requirements: Report of a joint FAO/WHO/ONU expert consultation, Roma, 2001 [Internet]. Food and Nutrition Technical Report Series 1 [citado 10 ago 2016]. Disponible en: http://tinyurl.com/jqtxdu6.

20. Consejo Nacional de Población. Indicadores de la dinámica demográfica 1990-2010 y de proyecciones de población 2010-2030 [Internet]. México: SEGOB; 2014 [citado may 2015]. Disponible en: http://tinyurl.com/gw7pnb9.

21. Instituto Nacional de Estadística y Geografía. Población, distribución por edad y sexo, pirámides de población [Internet]. INEGI [citado may 2015]. Disponible en: http://tinyurl.com/h5wfvmy.

22. World Health Organization. Energy and protein requirements: Report of a joint FAO/WHO/UNU expert consultation. Geneva: WHO; 1985.

23. Barquera $S$, Hernández-Barrera $L$, Tolentino ML, Espinosa J, Weng NS, River JA, Popkin BM. Energy intake from beverages is increasing among Mexican adolescents and adults. Journal of $\mathrm{Nu}-$ trition. 2008;138(12):2454-2461. doi: 10.3945/ jn.108.092163.

24. Fundación Heinrich Boll. Atlas de la carne: hechos y cifras sobre animales que comemos. México DF: Atlas Manufktur; 2014.
25. Pérez-Lizaur AB, Palacios-González B, CastroBecerra AL. Sistema mexicano de alimentos equivalentes. 3a ed. México: Fomento de Nutrición y Salud, Ogali; 2008.

26. Veraza J, (coord). Los peligros de comer en el capitalismo. México: Itaca; 2007.

27. Rosovsky H, Narváez A, Borges G, González L. Evolución del consumo per cápita de alcohol en México. Salud Mental. 1992;15(1):35-41.

28. Recio G. 2004. El nacimiento de la industria cervecera en México, 1880-1910 [Internet]. En: Segundo Congreso Nacional de Historia Económica. México: Facultad de Economía, UNAM [citado 29 sep 2015]. Disponible en: http://tinyurl. com/jlzlzqx.

29. Consejo de Investigación sobre Salud y Cerveza de México. La cerveza en México [Internet]. México. [citado 3 oct 2015]. Disponible en: http://tinyurl.com/hq2x2dv.

30. De la Fuente R, Medina-Mora ME. Las adicciones en México: El abuso de alcohol y los problemas relacionados. Salud Mental. 1987;10(2):3-13.

31. Dobson A, Porteous J, McElduff P, Alexander H. Dietary trends: estimates from food supply and survey data. European Journal of Clinical Nutrition. 1997;51(3):193-138.

32. Menéndez EL. Epidemiología Sociocultural: Propuestas y posibilidades. Región y Sociedad. 2008;20(Spec 2):5-50.

33. Contra los mitos en el consumo de azúcar. El Diario de Yucatán [Internet]. 23 may 2014 [citado 18 abr 2015]. Disponible en: http://tinyurl.com/ gmh2msg.

34. Arvizú-Martínez O, Polo-Oteyza E, Shamah-Levy T, (ed). Qué y cómo comemos los mexicanos [Internet]. México: Instituto Nacional de Salud Pública, Fundación Mexicana para la Salud; 2015 [citado 30 may 2015]. Disponible en: http:// tinyurl.com/j9jw5bs.

35. Instituto Nacional de Estadística y Geografía. Encuestas Nacionales de Ingresos y Gastos en los Hogares [Internet]. INEGI [citado 19 abr 2015]. Disponibles en: http://tinyurl.com/jpaasjc.

36. Organización de las Naciones Unidas para la Alimentación y la Agricultura. Ganadería bovina en América Latina: Escenario 2008-2009 y tendencias del sector [Internet]. Santiago de Chile: FAO; 2010 [citado 5 sep 2015]. Disponible en: http://tinyurl.com/h8wlump. 
37. Centro de Actividad Regional para el Consumo y la Producción Sostenible (CPRAC). La carne, un alimento caro en recursos [Internet]. Barcelona: SCP/RAC [citado 21 oct 2015]. Disponible en: http://tinyurl.com/zdyyot5.

38. Eshel G, Martin PA. Diet, energy and global warming. Earth Interactions. 2006;10:1-17.

39. Appendini K. De la milpa a los tortibonos: la restructuración de la política alimentaria en
México. 2a ed. México: El Colegio de México, Centro de Estudios Económicos: Instituto de Investigaciones de las Naciones Unidas para el Desarrollo Social; 2001.

40. El Semanario. México importa más de la mitad de sus alimentos: académica [Internet]. 26 feb 2015 [citado 9 ago 2016]. Disponible en: http:// tinyurl.com/zqg6ad2.

\section{FORMA DE CITAR}

Hernández Ramírez JC, Ortega Canto JE. El perfil general del excedente nutrimental en México en el periodo 19902013: un enfoque a partir del suministro energético de macronutrimentos y grupos de alimentos. Salud Colectiva. 2016;12(4):487-504. doi: 10.18294/sc.2016.925.

Recibido: 4 de abril de 2016 | Versión final: 24 de agosto de 2016 | Aprobado: 7 de octubre de 2016

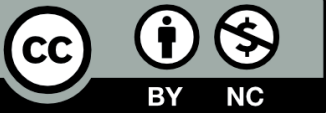

Este obra está bajo una licencia de Creative Commons Reconocimiento-NoComercial 4.0 Internacional. Reconocimiento - Permite copiar, distribuir y comunicar públicamente la obra. A cambio, se debe reconocer y citar al autor original. No Comercial - Esta obra no puede ser utilizada con finalidades comerciales, a menos que se obtenga el permiso.

http://dx.doi.org/10.18294/sc.2016.925 